International Journal of Pure and Applied Mathematics

Volume 86 No. 3 2013, 471-485

ISSN: 1311-8080 (printed version); ISSN: 1314-3395 (on-line version)

url: http://www.ijpam.eu

doi: http://dx.doi.org/10.12732/ijpam.v86i3.2

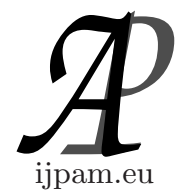

\title{
LC HELIX ON HYPERSURFACES IN \\ MINKOWSKI SPACE $\mathbb{E}_{1}^{n+1}$
}

\author{
Siddika Özkaldi Karakuş ${ }^{1}$, Ali Şenol ${ }^{2}$, Raheleh Ghadami ${ }^{3}$, Yusuf Yayli ${ }^{4}$ \\ ${ }^{1}$ Department of Mathematics \\ Faculty of Science \\ University of Bilecik Şeyh Edebali \\ Bilecik, TURKEY \\ ${ }^{2}$ Department of Mathematics \\ Faculty of Science \\ University of Cankiri Karatekin \\ Cankiri, TURKEY \\ ${ }^{3,4}$ Department of Mathematics \\ Faculty of Science \\ University of Ankara \\ Tandoğan, Ankara, TURKEY
}

\begin{abstract}
In this paper we give the definition of a generalized LC helix for a non-null curve lying on a hypersurface in $\mathbb{E}_{1}^{n+1}$ by using the Levi Civita's notion of parallel vector field. Also we give some basic properties and characterization of generalized LC helices.
\end{abstract}

AMS Subject Classification: 53C40, 53C50, 53B30

Key Words: LC helix, hypersurface, Minkowski space

\section{Introduction}

Helix is one of the most fascinating curves in science and nature. Scientists have

Received: February 10, 2013

(C) 2013 Academic Publications, Ltd.

$\S_{\text {Correspondence author }}$ url: www.acadpubl.eu 
long held a fascination, sometimes bordering on mystical obsession for helical structures in nature. Helices arise in nanosprings, carbon nanotubes, a-helices, DNA double and collagen triple helix, the double helix shape is commonly associated with DNA, since the double helix is structure of DNA.

A curve of constant slope or general helix in Euclidean 3 -space $\mathbb{E}^{3}$, is defined by the property that the tangent makes a constant angle with a fixed straight line (the axis of the general helix). A classical result stated by Lancret in 1802 and first proved by de Saint Venant in 1845 [14] is: A necessary and sufficient condition that a curve be a general helix is that the ratio of curvature to torsion be constant. If both of $k_{1}$ and $k_{2}$ are non-zero constants it is, of course, a general helix. It is known that straight line and circle are degenerate helix examples. $\left(k_{1}=0\right.$, if the curve is straight line and $k_{2}=0$, if the curve is a circle) [9].

The notion of a generalized helix can be generalized to higher dimensions in many ways. In [12] the same definition is proposed but in $\mathbb{E}^{n}$. In [7] the definition which was defined by Hayden is more restrictive: the fixed direction makes a constant angle with all the vectors of the Frenet frame. This definition only works in the odd dimensional case. Moreover, in the same reference, it is proved that the definition is equivalent to the fact that the ratios $\frac{k_{\mathrm{n}-1}}{k_{\mathrm{n}-2}}, \frac{k_{\mathrm{n}-3}}{k_{\mathrm{n}-4}}, \ldots, \frac{k_{2}}{k_{1}}$ being the curvatures, are constant. This statement is related with the Lancret Theorem for generalized helices in $\mathbb{E}^{3}$ (the ratio of torsion to curvature is constant). In [11] the curves in $\mathbb{E}^{n}$ for which all the ratios $\frac{k_{n}-1}{k_{n}-2}, \frac{k_{n-3}}{k_{n-4}}, \ldots, \frac{k_{2}}{k_{1}}$ are constant which was called ccr curves. In the same reference, it is shown that in the even dimensional case, a curve has constant curvature ratios if and only if its tangent indicatrix is a geodesic in the flat torus.

As a matter of fact, circular helix is the simplest three-dimensional spirals. One of the most interesting spiral example is $k-$ Fibonacci spirals. These curves appear naturally from studying the $k$-Fibonacci numbers $\left\{F_{k, n}\right\}_{n=0}^{\infty}$ and the related hyperbolic $k$-Fibonacci function. Fibonacci numbers and the related Golden Mean or Golden section appear very often in theoretical physics and physics of the high energy particles $[3,4]$. Three-dimensional, $k$-Fibonacci spirals was studied from a geometric point of view in [5].

Helices have been studied intensively by geometers and other scientists. In [2] some characterizations for a non-degenerate curve a to be a generalized as helix by using harmonic curvatures of the curve in n-dimensional Euclidean space $\mathbb{E}^{n}$. Morever, in [10] curvature conditions are given for $A W(k)$-type curves in Lorentzian space and shown that under what conditions $A W(k)$-type curves are helix.

The Lancert theorem was revisited and solved by M. Barros in 3-dimensional 
real space-forms by using Killing vector fields along curves. Also, in [1] M. Barros showed that a relation such as $\tau=b \kappa+a$ for a general helices in 3dimensional real space-form, where $a$ and $b$ are constant.

A helical curve is a curve in 3-dimensional space form $M^{3}(c)$ of constant curvature $c$ whose both curvature and torsion are non zero constants. It reduces to a Riemannian a circle or a geodesic [15].

Levi Civita's notion of parallellism (or parallel motion of a direction along a curve) is defined for the vector field along a curve located on a hypersurface. In this paper we give the definition of a generalized LC helix for a non-null curve lying on a hypersurface in $\mathbb{E}_{1}^{n+1}$ by using the Levi Civita's notion of parallel vector field. Also we give some basic properties and characterization of generalized LC helices. In particular, if the hypersurface which the non- null curve lying on is replaced by $\mathbb{E}_{1}^{n}$, then the definition of a helix on $\mathbb{E}_{1}^{n}$ coincides with the definition of generalized LC helix.

\section{Preliminaries}

Let $E_{1}^{n+1}$ be the $(n+1)$ dimensional pseudo-Euclidean space with index 1 endowed with the indefinite inner product given by

$$
g(x, y)=-x_{1} y_{1}+\sum_{i=2}^{n+1} x_{i} y_{i},
$$

where $x=\left(x_{1}, x_{2}, \cdots, x_{n+1}\right), y=\left(y_{1}, y_{2}, \cdots, y_{n+1}\right)$ is the usual coordinate system. Let $M$ be a hypersurface in $E_{1}^{n+1}$ and $p$ be a point on $M$ and $v \in T_{p} M$ a tangent vector. Then $v$ is said to be spacelike, timelike or null according to $g(v, v)>0, g(v, v)<0$, or $g(v, v)=0$ and $v \neq 0$, respectively. Notice that the vector $v=0$ is spacelike. The category into which a given tangent vector falls is called its causal character. These definitions can be generalized for curves as follows. A curve $\alpha$ on $M$ is said to be spacelike if all of its velocity vectors $\alpha$ are spacelike, similarly for timelike and null [1].

Let us recall from $[13,8]$ the definition of the Frenet frame and curvatures.

Let $M$ be a hypersurface in $E_{1}^{n+1}$ and $\alpha: I \subset \mathbb{R} \rightarrow M$ be non-null curve on $M$. A non-null curve $\alpha(s)$ is said to be a unit speed curve if $g\left(\alpha^{\prime}(s), \alpha^{\prime}(s)\right)=$ $\varepsilon_{0}$, ( $\varepsilon_{0}$ being +1 or -1 according to $\alpha$ is spacelike or timelike respectively). Let $\left\{V_{1}, V_{2}, \ldots, V_{n}\right\}$ be the moving Frenet frame along the unit speed curve $\alpha$, where $V_{i}(i=1,2, \ldots, n)$ denote $i$ th Frenet vector fields and $k_{i}$ be $i$ th curvature functions of the curve $(i=1,2, \ldots, n-1)$. Then the Frenet formulas are given 
by

$$
\begin{aligned}
\nabla_{V_{1}} V_{1} & =k_{1} V_{2}, \\
\nabla_{V_{1}} V_{i} & =-\varepsilon_{i-2} \varepsilon_{i-1} k_{i-1} V_{i-1}+k_{i} V_{i+1}, \quad 1<i<n \\
\nabla_{V_{1}} V_{n} & =-\varepsilon_{n-2} \varepsilon_{n-1} k_{n-1} V_{n-1}
\end{aligned}
$$

where $g\left(V_{i}, V_{i}\right)=\varepsilon_{i-1}$, and $\nabla$ is the Levi-Civita connection of $M$.

Let $M$ be a hypersurface in $E_{1}^{n+1}$ with the Levi-Civita connection $\nabla$ and suppose $\alpha: I \subset \mathbb{R} \rightarrow M$ is non-null curve on $M$. For any tangent vector $X$ at $\alpha(s)$ is said to be a Levi Civita's notion of parallel vector field on $M$ of a direction along a curve if $\nabla_{V_{1}} X=0$. Also, the Levi Civita's notion of parallel vector field has constant lenght [6].

\section{LC Helix on Hypersurfaces in Minkowski Space}

In this section we define LC helices on hypersurfaces in Minkowski space and we give some characterizations for LC helices on hypersurfaces in the same space.

Definition 1. Let $M$ be a hypersurface in $E_{1}^{n+1}$ and $\alpha: I \subset \mathbb{R} \rightarrow M$ be non-null curve on $M$. A non-null curve $\alpha(s)$ is said to be a generalized LC helix if there exists a Levi Civita's notion of parallel vector field $X$ on $M$ such that $g\left(V_{1}, X\right)$ is a constant function. Any line parallel this direction $X$ is called the axis of the LC generalized helix.

Definition 2. Let $M$ be a hypersurface in $E_{1}^{n+1}$ and non-null curve $\alpha$ : $I \subset \mathbb{R} \rightarrow M$ be a unit speed generalized LC helix. Harmonic curvatures of $\alpha$ is defined by $H_{i}: I \subset \mathbb{R} \rightarrow \mathbb{R}$,

$$
\begin{aligned}
& H_{1}=\varepsilon_{0} \varepsilon_{1} \frac{k_{1}}{k_{2}} \\
& H_{i}=\left(\varepsilon_{i-1} \varepsilon_{i} k_{i} H_{i-2}+\nabla_{V_{1}} H_{i-1}\right) \frac{1}{k_{i+1}}, \quad 2 \leq i \leq n-2,
\end{aligned}
$$

where $k_{1}, k_{2}, \ldots, k_{n-1}$ are curvatures functions of the curve $\alpha$ which are not necessarily constant.

Theorem 3. Let $M$ be a hypersurface in $E_{1}^{n+1}$ and $\alpha: I \subset \mathbb{R} \rightarrow M$ be a unit speed generalized $L C$ helix. Let $\left\{V_{1}, V_{2}, \ldots, V_{n}\right\},\left\{H_{1}, H_{2}, \ldots, H_{n-2}\right\}$ be denote the Frenet frame and the higher ordered harmonic curvatures of the curve, respectively. Then the following equations is holds

$$
g\left(V_{i+2}, X\right)=H_{i} g\left(V_{1}, X\right), \quad 1 \leq i \leq n-2,
$$


where $X$ is axis of the $L C$ generalized helix.

Proof. We use mathematical induction on $i$. Since $X$ is axis of the generalized LC helix $\alpha$, we get

$$
X=\lambda_{1} V_{1}+\lambda_{2} V_{2}+\ldots+\lambda_{n} V_{n}
$$

From the definition of generalized LC helix we have

$$
g\left(V_{1}, X\right)=\varepsilon_{0} \lambda_{1}
$$

By taking the derivative of (4) and applying the Frenet formulas twice, we obtain

$$
\begin{gathered}
g\left(V_{2}, X\right)=0, \\
g\left(V_{3}, X\right)=H_{1} g\left(V_{1}, X\right) .
\end{gathered}
$$

respectively. Hence it is shown that (3) is true for $i=1$. We now assume (3) is true for the first $i-1$. Then we have

$$
g\left(V_{i+1}, X\right)=H_{i-1} g\left(V_{1}, X\right)
$$

By taking the derivative of (5) and applying the Frenet formulas, we get

$$
-\varepsilon_{i-1} \varepsilon_{i} k_{i} g\left(V_{i}, X\right)+k_{i+1} g\left(V_{i+1}, X\right)=\nabla_{V_{1}} H_{i-1} g\left(V_{1}, X\right) .
$$

By using the our induction hypothesis, $g\left(V_{i}, X\right)=H_{i-2} g\left(V_{1}, X\right)$, we have

$$
\frac{1}{k_{i+1}}\left(\varepsilon_{i-1} \varepsilon_{i} k_{i} H_{i-2}+\nabla_{V_{1}} H_{i-1}\right) g\left(V_{1}, X\right)=g\left(V_{i+2}, X\right)
$$

it follows that

$$
g\left(V_{i+2}, X\right)=H_{i} g\left(V_{1}, X\right)
$$

Theorem 4. Let $M$ be a hypersurface in $E_{1}^{n+1}$ and non-null curve $\alpha$ : $I \subset \mathbb{R} \rightarrow M$ be a unit speed generalized $L C$ helix with Frenet vector fields $\left\{V_{1}, V_{2}, \ldots, V_{n}\right\}$, and harmonic curvatures $\left\{H_{1}, H_{2}, \ldots, H_{n-2}\right\}$. If $\alpha$ is generalized $L C$ helix, then $\sum_{i=1}^{n-2} \varepsilon_{i+1} H_{i}^{2}=c$, where $c$ is any constant. 
Proof. Let $\alpha$ be generalized LC helix with the arc length parameter $s$. Then by the definition we can assume that

$$
g\left(V_{1}, X\right)=\varepsilon_{0} \lambda_{1}
$$

for some non zero constant $\lambda_{1}$. By taking the derivative of (6) with respect to $s$ and applying the Frenet formulas we have

$$
g\left(k_{1} V_{2}, X\right)=0
$$

Since $k_{1} \neq 0$, then we have

$$
g\left(V_{2}, X\right)=0
$$

On the other hand, the fact that $X$ is axis of the LC generalized helix $\alpha$, we get

$$
\begin{aligned}
& X=\sum_{i=1}^{n} \lambda_{i} V_{i} \\
& X=\varepsilon_{0} g\left(V_{1}, X\right) V_{1}+\varepsilon_{2} g\left(V_{3}, X\right) V_{3}+\ldots+\varepsilon_{n-1} g\left(V_{n}, X\right) V_{n} .
\end{aligned}
$$

From Theorem(3) we can write

$$
X=\varepsilon_{0} g\left(V_{1}, X\right) V_{1}+g\left(V_{1}, X\right) \sum_{j=1}^{n-2} \varepsilon_{j+1} H_{j} V_{j+2},
$$

and notice that $X$ is a unit Levi Civita's notion of parallel vector field, we obtain

$$
\varepsilon_{0}\left(g\left(V_{1}, X\right)\right)^{2}+\left(g\left(V_{1}, X\right)\right)^{2} \sum_{j=1}^{n-2} \varepsilon_{j+1} H_{j}^{2}=1
$$

Thus we get

$$
\sum_{j=1}^{n-2} \varepsilon_{j+1} H_{j}^{2}=\frac{1-\varepsilon_{0} \lambda_{1}^{2}}{\lambda_{1}^{2}}
$$

This completes the proof.

Then we have the following corollary.

Corollary 5. If $X$ is axis of the $L C$ generalized helix $\alpha$ on $M$, then we can write

$$
X=\sum_{i=1}^{n} \lambda_{i} V_{i}
$$


By using the Theorem (3), we get

$$
X=g\left(V_{1}, X\right)\left(\varepsilon_{0} V_{1}+\sum_{j=1}^{n-2} \varepsilon_{j+1} H_{j} V_{j+2}\right)
$$

where $g\left(V_{1}, X\right)=\varepsilon_{0} \lambda_{1}=$ constant. Also

$$
\frac{W}{\|W\|}=\frac{\varepsilon_{0} V_{1}+\varepsilon_{2} H_{1} V_{3}+\ldots+\varepsilon_{n-1} H_{n-2} V_{n}}{\sqrt{\varepsilon_{0}+\varepsilon_{2} H_{1}^{2}+\ldots+\varepsilon_{n-1} H_{n-2}^{2}}}
$$

is a unit axis of the $L C$ generalized helix $\alpha$.

Theorem 6. Let $M$ be a hypersurface in $E_{1}^{n+1}$ and non-null curve $\alpha$ : $I \subset \mathbb{R} \rightarrow M$ be a unit speed curve with Frenet vector fields $\left\{V_{1}, V_{2}, \ldots, V_{n}\right\}$, and harmonic curvatures $\left\{H_{1}, H_{2}, \ldots, H_{n-2}\right\}$. Then $\alpha$ is a generalized LC helix if and only if $W=\varepsilon_{0} V_{1}+\varepsilon_{2} H_{1} V_{3}+\ldots+\varepsilon_{n-1} H_{n-2} V_{n}$ is Levi Civita's notion of parallel vector field.

Proof. Suppose that $\alpha$ is a generalized LC helix on $M$ and $X$ is axis of $\alpha$. From Corollary(5), we get

$$
X=\varepsilon_{0} \lambda_{1}\left(\varepsilon_{0} V_{1}+\varepsilon_{2} H_{1} V_{3}+\ldots+\varepsilon_{n-1} H_{n-2} V_{n}\right) .
$$

By taking the derivative of (7), and using the definition of the Levi Civita's notion of parallel vector field we can easily obtain

$$
\nabla_{V_{1}} W=0
$$

Thus $W$ is Levi Civita's notion of parallel vector field.

Conversely, since $W$ is Levi Civita's notion of parallel vector field then $\|W\|=$ constant. We consider the normalisation of the Levi Civita's notion of parallel vector field as follows

$$
X=\frac{1}{\|W\|} W
$$

Therefore we have

$$
g\left(V_{1}, X\right)=\frac{1}{\|W\|}=\text { constant. }
$$

Thus $\alpha$ is a generalized LC helix.

Theorem 7. Let $M$ be a hypersurface in $E_{1}^{n+1}$, and $\alpha$ be a geodesic on $M$. Then $\alpha$ is a generalized $L C$ helix. 
Proof. Let $\alpha$ be a geodesic on $M$ with the arc length parameter $s$ and suppose that $X=V_{1}$. From the Gauss formula we have

$$
\begin{aligned}
\nabla_{V_{1}} X & =\nabla_{V_{1}}^{0} X-<\nabla_{V_{1}}^{0} X, N>N \\
& =\frac{d^{2} \alpha}{d s^{2}}-<\frac{d^{2} \alpha}{d s^{2}}, N>N
\end{aligned}
$$

where $\nabla^{0}$ is the Levi-Civita connection of $E_{1}^{n+1}$ and $N$ is normal unit vector field on $M$. By the definition of geodesic we have $\frac{d^{2} \alpha}{d s^{2}}=\lambda N$ for some constant $\lambda$. Hence

$$
\nabla_{V_{1}} X=0
$$

and clearly $g\left(X, V_{1}\right)=g\left(V_{1}, V_{1}\right)=\varepsilon_{0}$ is constant. Thus $\alpha$ is a generalized LC helix.

From now, let us consider $M$ is a hypersurface in $E_{1}^{4}$ and $\alpha: I \subset \mathbb{R} \rightarrow$ $M$ be a non-null curve with Frenet vector fields $\left\{V_{1}, V_{2}, V_{3}\right\}$ and curvatures $\left\{k_{1}, k_{2}\right\}$. Then the Frenet formulas are given by

$$
\begin{aligned}
& \nabla_{V_{1}} V_{1}=k_{1} V_{2}, \\
& \nabla_{V_{1}} V_{2}=-\varepsilon_{0} \varepsilon_{1} k_{1} V_{1}+k_{2} V_{3}, \\
& \nabla_{V_{1}} V_{3}=-\varepsilon_{1} \varepsilon_{2} k_{2} V_{2} .
\end{aligned}
$$

Theorem 8. Let $M$ be a hypersurface in $E_{1}^{4}$, and $\alpha$ be a regular curve on $M$. Then $\alpha$ satisfies the following equation

$$
\begin{aligned}
\nabla_{V_{1}}^{3} V_{1}-\left(2 \frac{k_{1}^{\prime}}{k_{1}}+\frac{k_{2}^{\prime}}{k_{2}}\right) & \nabla_{V_{1}}^{2} V_{1} \\
+ & {\left[-\frac{k_{1}^{\prime \prime}}{k_{1}}+\frac{k_{1}^{\prime}}{k_{1}} \frac{k_{2}^{\prime}}{k_{2}}+2\left(\frac{k_{1}^{\prime}}{k_{1}}\right)^{2}+\varepsilon_{0} \varepsilon_{1} k_{1}^{2}+\varepsilon_{1} \varepsilon_{2} k_{2}^{2}\right] } \\
& \nabla_{V_{1}} V_{1}+\varepsilon_{0} \varepsilon_{1} k_{1} k_{2}\left(\frac{k_{1}}{k_{2}}\right)^{\prime} V_{1}=0 .
\end{aligned}
$$

Theorem 9. Let $M$ be a hypersurface in $E_{1}^{4}$, and $\alpha$ be a regular curve on $M$. Then $\alpha$ is a generalized $L C$ helix if and only if $\frac{k_{1}}{k_{2}}$ is constant.

Proof. Let $M$ be a hypersurface in $E_{1}^{4}$, and $\alpha$ is a generalized LC helix on $M$. Without loss of generality, assume $\alpha$ has unit speed. By the definition of LC helix, there exists a Levi Civita's notion of parallel vector field $X$ such that

$$
g\left(V_{1}, X\right)=c_{1}
$$


for some non zero constant $c_{1}$. By taking the derivative of (9) and applying the Frenet formulas

$$
\begin{array}{r}
g\left(\nabla_{V_{1}} V_{1}, X\right)=0 \\
g\left(k_{1} V_{2}, X\right)=0 .
\end{array}
$$

Since $k_{1} \neq 0$, then we get

$$
g\left(V_{2}, X\right)=0
$$

Now, $X$ is perpendicular to $V_{2}$, so

$$
X=a V_{1}+b V_{3}
$$

for some non zero function $a, b$. Because $X$ is a unit Levi Civita's notion of parallel vector field, $\nabla_{V_{1}} X=0$. By taking the derivative of (10), and applying the Frenet formulas we have

$$
0=a^{\prime} V_{1}+\left(a k_{1}-\varepsilon_{1} \varepsilon_{2} k_{2} b\right) V_{2}+b^{\prime} V_{3}
$$

Since $\left\{V_{1}, V_{2}, V_{3}\right\}$ are linearly independent we have

$$
\begin{gathered}
a^{\prime}=0, \\
a k_{1}-\varepsilon_{1} \varepsilon_{2} k_{2} b=0, \\
b^{\prime}=0 .
\end{gathered}
$$

Hence

$$
\frac{k_{1}}{k_{2}}=\varepsilon_{1} \varepsilon_{2} \frac{b}{a}=\text { constant. }
$$

Now suppose that $\frac{k_{1}}{k_{2}}$ is constant. We can choose $\frac{k_{1}}{k_{2}}=\varepsilon_{1} \varepsilon_{2} c$, for some non zero constant $c$ and define

$$
X=\frac{1}{c} V_{1}+V_{3}
$$

to get

$$
\nabla_{V_{1}} X=\frac{1}{c} k_{1} V_{2}-\varepsilon_{1} \varepsilon_{2} k_{2} V_{2}=0 .
$$

Hence, $X$ is a Levi Civita's notion of parallel vector field and clearly $g\left(X, V_{1}\right)=$ $\frac{1}{c}$ is constant. Thus $\alpha$ is a generalized LC helix. 
Example 10. Timelike LC Helix on $S_{1}^{3}$.

$$
\alpha(t)=\left(\frac{2}{3} \cos 3 t, \frac{2}{3} \sin 3 t, \frac{\sqrt{5}}{3} \cosh 3 t, \frac{\sqrt{5}}{3} \sinh 3 t\right) .
$$

is a unit speed curve on $S_{1}^{3}$. The Frenet vector fields $\left\{V_{1}, V_{2}, V_{3}\right\}$ and the curvatures $k_{1}$ and $k_{2}$, are obtained as follows:

$$
\begin{gathered}
V_{1}=(-2 \sin 3 t, 2 \cos 3 t, \sqrt{5} \sinh 3 t, \sqrt{5} \cosh 3 t), \\
V_{2}=\left(-\frac{\sqrt{5}}{3} \cos 3 t,-\frac{\sqrt{5}}{3} \sin 3 t, \frac{2}{3} \cosh 3 t, \frac{2}{3} \sinh 3 t\right), \\
V_{3}=(\sqrt{5} \sin 3 t,-\sqrt{5} \cos 3 t,-2 \sinh 3 t,-2 \cosh 3 t), \\
k_{1}=4 \sqrt{5}, \quad k_{2}=9 .
\end{gathered}
$$

Then $\alpha(t)$ is a timelike LC helix with the axis

$$
X=\left(\frac{\sqrt{5}}{10} \sin 3 t,-\frac{\sqrt{5}}{10} \cos 3 t, \frac{1}{4} \sinh 3 t, \frac{1}{4} \cosh 3 t\right) .
$$

Theorem 11. Let $M$ be a hypersurface in $E_{1}^{4}$, and $\alpha$ be a regular curve on $M$. Then $\alpha$ is a generalized $L C$ helix if and only if the equation

$$
\begin{aligned}
\nabla_{V_{1}}^{3} V_{1}-\left(3 \frac{k_{1}^{\prime}}{k_{2}}\right) \nabla_{V_{1}}^{2} V_{1} & \\
& +\left[-\frac{k_{1}^{\prime \prime}}{k_{1}}+3\left(\frac{k_{1}^{\prime}}{k_{1}}\right)^{2}+\varepsilon_{0} \varepsilon_{1} k_{1}^{2}+\varepsilon_{1} \varepsilon_{2} k_{2}^{2}\right] \nabla_{V_{1}} V_{1}=0
\end{aligned}
$$

holds for $\alpha$.

Proof. Let $\alpha$ be a generalized LC helix. Since $\alpha$ is a regular curve, it satisfies the equation in (11). Morever, from Theorem(9)we have $\frac{k_{1}}{k_{2}}$ is constant. Thus

$$
\begin{array}{r}
-\left(2 \frac{k_{1}^{\prime}}{k_{1}}+\frac{k_{2}^{\prime}}{k_{2}}\right)=-3 \frac{k_{1}^{\prime}}{k_{1}}, \\
{\left[-\frac{k_{1}^{\prime \prime}}{k_{1}}+\frac{k_{1}^{\prime}}{k_{1}} \frac{k_{2}^{\prime}}{k_{2}}+2\left(\frac{k_{1}^{\prime}}{k_{1}}\right)^{2}+\varepsilon_{0} \varepsilon_{1} k_{1}^{2}+\varepsilon_{1} \varepsilon_{2} k_{2}^{2}\right]}
\end{array}
$$




$$
\begin{gathered}
=-\frac{k_{1}^{\prime \prime}}{k_{1}}+3\left(\frac{k_{1}^{\prime}}{k_{1}}\right)^{2}+\varepsilon_{0} \varepsilon_{1} k_{1}^{2}+\varepsilon_{1} \varepsilon_{2} k_{2}^{2}, \\
\varepsilon_{0} \varepsilon_{1} k_{1} k_{2}\left(\frac{k_{1}}{k_{2}}\right)^{\prime}=0,
\end{gathered}
$$

then setting these equalities in (8) we obtain equation (11).

Conversely assume that the regular curve $\alpha$ satisfies the equality in . Since $\alpha$ is a regular curve it also satisfies the equality in (11). Substracting (11) from (8), we get

$$
X \nabla_{V_{1}}^{2} V_{1}+Y \nabla_{V_{1}} V_{1}+\varepsilon_{0} \varepsilon_{1} Z V_{1}=0
$$

where

$$
\begin{aligned}
X & =\frac{k_{1}^{\prime}}{k_{1}}-\frac{k_{2}^{\prime}}{k_{2}}, \\
Y & =\frac{k_{1}^{\prime}}{k_{1}} \frac{k_{2}^{\prime}}{k_{2}}-\left(\frac{k_{1}^{\prime}}{k_{1}}\right)^{2}, \\
Z & =k_{1} k_{2}\left(\frac{k_{1}}{k_{2}}\right)^{\prime} .
\end{aligned}
$$

Setting the equations

$$
\begin{gathered}
\nabla_{V_{1}} V_{1}=k_{1} V_{2} \\
\nabla_{V_{1}}^{2} V_{1}=\left(-\varepsilon_{0} \varepsilon_{1} k_{1}^{2}\right) V_{1}+k_{1}^{\prime} V_{2}+k_{1} k_{2} V_{3},
\end{gathered}
$$

in (12), we obtain

$$
X\left(-\varepsilon_{0} \varepsilon_{1} k_{1}^{2} V_{1}+k_{1}^{\prime} V_{2}+k_{1} k_{2} V_{3}\right)+Y k_{1} V_{2}+\varepsilon_{0} \varepsilon_{1} Z V_{1}=0
$$

and

$$
\left(-\varepsilon_{0} \varepsilon_{1} k_{1}^{2} X+\varepsilon_{0} \varepsilon_{1} Z\right) V_{1}+\left(X k_{1}^{\prime}+Y k_{1}\right) V_{2}+\left(k_{1} k_{2} X\right) V_{3}=0 .
$$

Since $\left\{V_{1}, V_{2}, V_{3}\right\}$ are linearly independent we have

$$
\begin{gathered}
-\varepsilon_{0} \varepsilon_{1} k_{1}^{2} X+\varepsilon_{0} \varepsilon_{1} Z=0, \\
X k_{1}^{\prime}+Y k_{1}=0, \\
k_{1} k_{2} X=0 .
\end{gathered}
$$

Hence

$$
\left(\frac{k_{1}}{k_{2}}\right)^{\prime}=0 .
$$


Solution of this equation leads to

$$
\frac{k_{1}}{k_{2}}=\text { constant }
$$

Thus the regular curve $\alpha$ is a generalized LC helix.

Theorem 12. Let $M$ be a hypersurface in $E_{1}^{4}$, and $\alpha$ be a regular curve on $M$. If $\alpha$ is a cyclic LC helix (i.e. $k_{1}=$ constant, $k_{2}=$ constant) then

$$
\nabla_{V_{1}}^{3} V_{1}+\left(\varepsilon_{0} \varepsilon_{1} k_{1}^{2}+\varepsilon_{1} \varepsilon_{2} k_{2}^{2}\right) \nabla_{V_{1}} V_{1}=0
$$

Proof. The proof is straight forward from Theorem(11).

Theorem 13. Let $M$ be a hypersurface in $E_{1}^{4}$ and $\alpha: I \subset \mathbb{R} \rightarrow M$ be a unit speed curve with Frenet vector fields $\left\{V_{1}, V_{2}, V_{3}\right\}$. Then $\alpha$ is a generalized $L C$ helix if and only if $\operatorname{det}\left(\nabla_{V_{1}} V_{3}, \nabla_{V_{1}}^{2} V_{3}, \nabla_{V_{1}}^{3} V_{3}\right)=0$.

Proof. Let $\alpha$ be a generalized LC helix. From the Frenet formulas we have

$$
\nabla_{V_{1}} V_{3}=-\varepsilon_{1} \varepsilon_{2} k_{2} V_{2}
$$

By taking the derivative of (13) and applying the Frenet formulas twice, we get

$$
\begin{gathered}
\nabla_{V_{1}}^{2} V_{3}=-\varepsilon_{1} k_{1} k_{2} V_{1}-\varepsilon_{1} \varepsilon_{2} k_{2}^{\prime} V_{2}-\varepsilon_{1} \varepsilon_{2} k_{2}^{2} V_{3} \\
\nabla_{V_{1}}^{2} V_{3}=\left(-\varepsilon_{1} k_{1}^{\prime} k_{2}-2 \varepsilon_{1} k_{1} k_{2}^{\prime}\right) V_{1}+\left(-\varepsilon_{1} k_{1}^{2}-\varepsilon_{1} \varepsilon_{2} k_{2}^{\prime \prime}+k_{2}^{3}\right) V_{2}+\left(-3 \varepsilon_{1} \varepsilon_{2} k_{2} k_{2}^{\prime}\right)
\end{gathered}
$$
respectively.

A straightforward computation leads to the following

$$
\operatorname{det}\left(\nabla_{V_{1}} V_{3}, \nabla_{V_{1}}^{2} V_{3}, \nabla_{V_{1}}^{3} V_{3}\right)=\varepsilon_{1} k_{2}^{5}\left(\frac{k_{1}}{k_{2}}\right)^{\prime}
$$

Since $\alpha$ is a general LC helix, then $\frac{k_{1}}{k_{2}}$ is constant, from which we have

$$
\operatorname{det}\left(\nabla_{V_{1}} V_{3}, \nabla_{V_{1}}^{2} V_{3}, \nabla_{V_{1}}^{3} V_{3}\right)=0 \text {. }
$$

Conversely, we assume that the equation $\operatorname{det}\left(\nabla_{V_{1}} V_{3}, \nabla_{V_{1}}^{2} V_{3}, \nabla_{V_{1}}^{3} V_{3}\right)=0$ holds, we easily obtain that $\frac{k_{1}}{k_{2}}$ is a constant function, then from Theorem (9) we have a is generalized LC helix, which concludes the proof.

Theorem 14. Let $M$ be a hypersurface in $E_{1}^{4}$ and $\alpha: I \subset \mathbb{R} \rightarrow M$ be a unit speed curve with Frenet vector fields $\left\{V_{1}, V_{2}, V_{3}\right\}$. Then $\alpha$ is a generalized $L C$ helix if and only if $\operatorname{det}\left(\nabla_{V_{1}}^{2} \alpha, \nabla_{V_{1}}^{3} \alpha, \nabla_{V_{1}}^{4} \alpha\right)=0$. 
Proof. Let $\alpha$ be a generalized LC helix. From the definition of Frenet frame

$$
\nabla_{V_{1}} \alpha=V_{1}
$$

Thus

$$
\nabla_{V_{1}}\left(\nabla_{V_{1}} \alpha\right)=\nabla_{V_{1}} V_{1}=k_{1} V_{2}
$$

By taking the derivative of (14) and applying the Frenet formulas twice, we get

$$
\begin{gathered}
\nabla_{V_{1}}\left(\nabla_{V_{1}}^{2} \alpha\right)=\nabla_{V_{1}}^{2} V_{1}=\left(-\varepsilon_{0} \varepsilon_{1} k_{1}^{2}\right) V_{1}+k_{1}^{\prime} V_{2}+k_{1} k_{2} V_{3}, \\
\nabla_{V_{1}}\left(\nabla_{V_{1}}^{3} \alpha\right)=\nabla_{V_{1}}^{3} V_{1}=A V_{1}+B V_{2}+C V_{3},
\end{gathered}
$$

respectively, where $A=-3 \varepsilon_{0} \varepsilon_{1} k_{1} k_{1}^{\prime}, B=-\varepsilon_{0} \varepsilon_{1} k_{1}^{3}+k_{1}^{\prime \prime}-\varepsilon_{1} \varepsilon_{2} k_{1} k_{2}^{2}, C=2 k_{1}^{\prime} k_{2}+$ $k_{1} k_{2}^{\prime}$.

A straightforward computation leads to the following

$$
\operatorname{det}\left(\nabla_{V_{1}}^{2} \alpha, \nabla_{V_{1}}^{3} \alpha, \nabla_{V_{1}}^{4} \alpha\right)=\varepsilon_{0} \varepsilon_{1} k_{1}^{5}\left(\frac{k_{2}}{k_{1}}\right)^{\prime} .
$$

Since $\alpha$ is a general LC helix, then $\frac{k_{1}}{k_{2}}$ is constant, from which we have

$$
\operatorname{det}\left(\nabla_{V_{1}}^{2} \alpha, \nabla_{V_{1}}^{3} \alpha, \nabla_{V_{1}}^{4} \alpha\right)=0 .
$$

Conversely, we assume that the equation $\operatorname{det}\left(\nabla_{V_{1}}^{2} \alpha, \nabla_{V_{1}}^{3} \alpha, \nabla_{V_{1}}^{4} \alpha\right)=0$ holds, we easily obtain that $\frac{k_{1}}{k_{2}}$ is a constant function, then from Theorem (9) we have a is generalized LC helix, which concludes the proof.

\section{Conclusion}

Helix is one of the most fascinating curves in science and nature. Helices arise in nanosprings, carbon nanotubes, a-helices, DNA double and collagen triple helix. Due to these properties, helix has played a very important role in the study of scientist.

Lorentzian geometry helps to bridge the gap between modern differential geometry and the mathematical physics of general relativity by giving an invariant treatment of Lorentzian geometry.

In this paper we give the definition of a generalized LC helix for a nonnull curve lying on a hypersurface in $\mathbb{E}_{1}^{n+1}$ by using the Levi Civita's notion of parallel vector field. In particular, if the hypersurface which the non- null curve lying on is replaced by $\mathbb{E}_{1}^{n}$, then the definition of a helix on $\mathbb{E}_{1}^{n}$ coincides with the definition of generalized LC helix. 


\section{References}

[1] M. Barros, General helices and a theorem of Lancert, Proc. AMS, 125 (1997).

[2] CaIlarslan K. mcı Ç, L. Kula, H.H. Hacısalihoğlu, Harmonic curvatures and generalized helices in $\mathbb{E}^{n}$, Chaos, Solitons $\mathscr{E}$ Fractals (2007), doi: 10.1016/j.chaos.2007.11.001.

[3] M.S. El Naschie, Notes on superstrings and the infinite sums of Fibonacci and Lucas numbers, Chaos, Solitons 85 Fractals, 12, No. 10 (2001), 19371940.

[4] M.S. El Naschie, Experimental and theoretical arguments for the number and mass of the Higgs particles, Chaos, Solitons 83 Fractals, 23 (2005); 1091-1098.

[5] S. Falcon, A. Plaza, On the three-dimensional $k$-Fibonacci spirals, Chaos, Solitons 83 Fractals (2007), doi: 10.1016/j.chaos2007.02.09.

[6] H.H. Hacisalihoglu, Diferensiyel Geometri, Ankara University Faculty of Science Press (1993).

[7] H.A. Hayden, On a general helix in a Riemannian $n$-space, Proc. London Math. Soc., 32, No. 2 (1931), 37-45.

[8] K. İlarslan, Some Special Curves on Non-Euclidean Manifolds, Ph.D. Thesis, Ankara University, Graduate School of Natural and Applied Sciences (2002).

[9] W. Kuhnel, Differential Geometry: Curves-Surfaces-Manifolds, Wiesbaden, Braunchweig (1999).

[10] M. Külahcı, M. Bektaş, M. Ergüt, On harmonic curvatures of a Frenet curve in Lorentzian space, Chaos, Solitons 8 Fractals (2008), doi: 10.1016/j.chaos.2008.07.013.

[11] J. Monterde, Curves with constant curvature ratios, ArXiv: math.DG/0412323.

[12] M.C. Romero-Fuster, E. Sanabria-Codesal, Generalized helices, twistings and flattenings of curves in n-space, In: 10-th School on Differential Geometry, Portuguese, Belo Horizonte (1998); Math. Contemp., 17 (1999), $67-80$. 
[13] H.H. Song, On proper helix in pseudo-Riemannian submanifolds, J.Geom., 91 (2008), 150-168.

[14] D.J. Struik, Lectures on Classical Differential Geometry, New York, Dover (1988).

[15] M. Tamura, Surfaces which contain helical geodesics in the 3-sphere, Mem. Fac. Sci. Eng. Shimane Unıv. Series B: Mathematical Science, 37 (2004), 59-65. 
